East African Medical Journal Vol. 86 No. 8 August 2009

SPORADIC PAEDIATRIC DIARRHOEAL ILLNESS IN URBAN AND RURAL SITES IN NYANZA PROVINCE, KENYA

M. E. Beatty, MD., MPH, Enteric Diseases Epidemiology Branch, Division of Food borne, Bacterial and Mycotic Diseases, National Center for Zoonotic, Vectorborne, and Enteric Diseases, Centers for Disease Control and Prevention, Atlanta GA and Epidemic Intelligence Service, Office of Workforce and Career Development, Centers for Disease Control and Prevention, Atlanta GA, J. B. Ochieng, Center for Global Health Research, Kenya Medical Research Institute, Kisumu, Kenya, W. Chege, MD., Enteric Diseases Epidemiology Branch, Division of Food borne, Bacterial and Mycotic Diseases, National Center for Zoonotic, Vectorborne, and Enteric Diseases, Centers for Disease Control and Prevention, Atlanta GA and Epidemic Intelligence Service, Office of Workforce and Career Development, Centers for Disease Control and Prevention, Atlanta GA, L. Kumar, Enteric Diseases Laboratory Branch, Division of Foodborne, Bacterial and Mycotic Diseases, National Center for Zoonotic, Vectorborne, and Enteric Diseases, Centers for Disease Control and Prevention, Atlanta GA, G. Okoth, Center for Global Health Research, Kenya Medical Research Institute, Kisumu, Kenya, R. L. Shapiro, MD., Enteric Diseases Epidemiology Branch, Division of Food borne, Bacterial and Mycotic Diseases, National Center for Zoonotic, Vectorborne, and Enteric Diseases, Centers for Disease Control and Prevention, Atlanta GA and Epidemic Intelligence Service, Office of Workforce and Career Development, Centers for Disease Control and Prevention, Atlanta GA, J. G. Wells, M. B. Parsons, C. Bopp, Enteric Diseases Laboratory Branch, Division of Foodborne, Bacterial and Mycotic Diseases, National Center for Zoonotic, Vectorborne, and Enteric Diseases, Centers for Disease Control and Prevention, Atlanta GA, T. Chiller, MD., Enteric Diseases Epidemiology Branch, Division of Food borne, Bacterial and Mycotic Diseases, National Center for Zoonotic, Vectorborne, and Enteric Diseases, Centers for Disease Control and Prevention, Atlanta GA, Epidemic Intelligence Service, Office of Workforce and Career Development, Centers for Disease Control and Prevention, Atlanta GA, J. Vulule, MD., Center for Global Health Research, Kenya Medical Research Institute, Kisumu, Kenya, E. Mintz, MD., MPH., Enteric Diseases Epidemiology Branch, Division of Food borne, Bacterial and Mycotic Diseases, National Center for Zoonotic, Vectorborne, and Enteric Diseases, Centers for Disease Control and Prevention, Atlanta GA, L. Slutsker, MD., MPH, Enteric Diseases Epidemiology Branch, Division of Food borne, Bacterial and Mycotic Diseases, National Center for Zoonotic, Vectorborne, and Enteric Diseases, Centers for Disease Control and Prevention, Atlanta GA, Center for Global Health Research, Kenya Medical Research Institute, Kisumu, Kenya and Division of Parasitic Diseases, National Center for Zoonotic, Vectorborne, and Enteric Diseases, Centers for Disease Control and Prevention, Atlanta GA and J. T. Brooks, MD., Enteric Diseases Epidemiology Branch, Division of Food borne, Bacterial and Mycotic Diseases, National Center for Zoonotic, Vectorborne, and Enteric Diseases, Centers for Disease Control and Prevention, Atlanta GA and Epidemic Intelligence Service, Office of Workforce and Career Development, Centers for Disease Control and Prevention, Atlanta GA

Request for reprints to: Dr. M. E. Beatty, Pediatric Dengue Vaccine Initiative International Vaccine Institute SNU Research Park, San 4-8 Bongcheon-7 -dong Kwanak-gu, Seoul, Korea 151-919

\title{
SPORADIC PAEDIATRIC DIARRHOEAL ILLNESS IN URBAN AND RURAL SITES IN NYANZA PROVINCE, KENYA
}

M.E. BEATTY, J. B. OCHIENG, W. CHEGE, L. KUMAR, G. OKOTH, R. L. SHAPIRO, J. G. WELLS, M. B. PARSONS, C. BOPP, T. CHILLER, J. VULULE, E. MINTZ, L. SLUTSKER, and J. T. BROOKS

\begin{abstract}
Objective: Investigate differences in the infectious aetiology, health seeking behaviour, and provider practices with regard to diarrhoeal illness among children presenting to urban versus rural clinics in Western Kenya.

Design: Laboratory-based, passive surveillance.

Setting: The urban portion of the study was conducted at the paediatric outpatient clinic of Nyanza Provincial Hospital in Kisumu. The rural portion of the study was conducted at four outpatient clinics in the Asembo Bay community approximately 20 kilometers west of Kisumu.

Subjects: Children aged less than five years presenting to medical facilities for the treatment of diarrhoea from October 2001-October 2003 at the urban site and May 1997-April 2003 for the rural sites.

Results: Among the 1303 urban and 1247 rural specimens collected, $24 \%$ of specimens yielded a bacterial pathogen ( $24 \%$ urban, $25 \%$ rural). Campylobacter was the predominant bacterial pathogen (17\% urban, $15 \%$ rural), followed by Shigella and nontyphoidal Salmonella (both $4 \%$ urban and $5 \%$ rural). In both communities, susceptibilities of these pathogens to the most commonly prescribed antibiotics was low $(\leq 50 \%) ; 70 \%$ of all
\end{abstract}




\begin{abstract}
episodes of diarrhoea were prescribed antibiotic treatment. Urban health practitioners prescribed fewer antibiotics, chose drugs more likely to be effective, and were more likely to prescribe oral rehydration therapy for bloody diarrhoea.

Conclusion: Most characteristics of diarrhoeal disease and their causes were similar in paediatric patients presenting to urban and rural clinics. Urban providers were more compliant with WHO recommendations.
\end{abstract}

\section{INTRODUCTION}

Diarrhoeal disease remains a major cause of morbidity and mortality in developing countries (1). Few systematic analyses have been published comparing aetiologic agents of diarrhoea and associated health practices in urban and rural communities in subSaharan Africa (2). Research is warranted to describe and understand differences between urban and rural communities within the same region given the potential differences in exposures and in the extent of training and experience of health care providers in each community. We compared paediatric diarrhoeal disease surveillance data collected from urban and rural sites in western Kenya to explore differences in bacterial aetiologies, antimicrobial resistance patterns, and health care practices.

\section{MATERIALS AND METHODS}

Study site: The study was conducted in Kenya's Nyanza Province, which borders Lake Victoria in western Kenya. The urban portion of the study was conducted at the paediatric outpatient clinic of Nyanza Provincial Hospital in Kisumu, Kenya's third largest city (population 322,724) (3); the hospital is a public government facility that serves primarily residents of Kisumu. The rural portion of the study was conducted at four outpatient clinics (two government-supported located in the villages of Ongielo and Abidha, two privately supported located in Lwak and Rarieda, later Sarididi) in the Asembo Bay community, approximately 20 kilometers west of Kisumu. Of the privately supported clinics, one was the clinic associated with a mission hospital while the other was a private solo provider. Asembo Bay is an area composed of 79 villages organised as loose conglomerates of family compounds separated by garden plots, grazing land and streams. Fishing, raising cattle, and subsistence farming are the principal occupations. In 1996, the population was 55,000 spread out over an area of an area of $200 \mathrm{~km}^{2}$ $(2,4,5)$.

Surveillance and specimen collection: We conducted clinic-based diarrhoeal surveillance with laboratory diagnosis at the urban clinic from October 18, 2001 through October 17,2003. Each weekday, the first five children aged $\leq 5$ years who presented to the clinic with a chief complaint of diarrhoeal illness were enrolled following informed consent. Because of the high patient volume at the urban site, only two years were required to obtain a sample size comparable to the rural sites. However, we conducted surveillance using the same methods at the rural clinics from May 1,1997 through April 30, 2003. During this period, every eligible resident of the study area presenting with the chief complaint of diarrhoeal illness at the rural clinics was offered an opportunity to enroll in the study. Data from the rural surveillance study have been previously reported $(5,6)$; we report here observations and analyses of data for children aged $\leq 5$ years not previously presented.

We defined diarrhoea as $\geq 3$ bowel movements in any 24-hour period within the preceding five days. We characterised diarrhoea as bloody if blood was visible in the specimen or the caretaker reported seeing blood in the patient's stool. Non-bloody diarrhoea was categorised as watery or mucoid, or if not described, uncharacterised.

For each enrolled child, we collected demographic information and medical histories including symptoms, medications taken before the visit, medications prescribed at the visit, and a stool specimen (either whole stool or a Dacron ${ }^{\circledR}$ rectal swab). Rectal swabs and swabs of whole stool specimens were placed in Cary-Blair transport medium, refrigerated at $4-8^{\circ} \mathrm{C}$, and transported within 48 hours of collection to the KEMRI/CDC Microbiology Laboratory.

Laboratory procedures: All stool specimens were cultured for Shigella, Salmonella, Vibrio and Campylobacter species. Pathogens were isolated, identified, and serotyped by standard methods. We determined antimicrobial susceptibilities of Shigella, Salmonella and Vibrio cholerae isolates to ampicillin, amoxicillin-clavulanate, ceftriaxone, chloramphenicol, ciprofloxacin, gentamicin, kanamicin, nalidixic acid, streptomycin, sulfisoxazole, tetracycline, and trimethoprimsulfamethoxazole (TMP/SMX) by the Kirby-Bauer disk diffusion method (7). We tested a randomly selected subset of Campylobacter isolates using the E-Test ${ }^{\circledR}$ method (AB Biodisk, Piscataway, NJ) for susceptibility to chloramphenicol, ciprofloxacin, clindamycin, erythromycin, cephalothin, nalidixic acid, tetracycline, and TMP/SMX (8). Serotypes 
of S. dysenteriae, S.flexneri, and all Salmonella were determined by standard methods using commercial antisera (9).

Statistical methods: We conducted statistical analyses using STATA version 7.0 (STATACorporation, College Station, TX). We calculated Pearson's chi-square, odds ratios (OR), and their exact 95\% confidence intervals $(95 \% \mathrm{CI})$. We report the mean of continuous data and tested for normality. Finding the data not normal, we report interquartile ranges and compared the distributions using the Mann-Whitney test. We applied linear regression analysis to examine linear relationships between pathogen isolation rates and patient age; we report the slope of the regression line $(\beta)$, its $95 \% \mathrm{CI}$, and the P-value for the association. Age-specific isolation rates were calculated using 6-month age categories (0-5 months, 6-11 months, 12-17 months). Noting that Campylobacter isolation rates peaked among 6-12 month old children, we further examined Campylobacter isolation rates for the children aged $\leq 12$ months by one-month age categories. We used logistic regression for all comparisons between urban and rural communities in order to control for differences in age distribution. We also controlled for the significantly greater number of unserotyped Salmonella from the urban site using logistic regression. To compare treatment patterns for dysentery (diarrhoeal stool containing blood), we developed a regression model that included prescription of any of the following for patients with bloody diarrhoea: oral rehydration solution (ORS), intravenous (IV) fluid, and antibiotics with Gramnegative rod (GNR) activity. When the analysis was restricted to only the data collected during the 18 month period of overlap in which the study was conducted at both the urban and rural sites, similar results were obtained but the smaller sample size and consequent loss of power led to a loss of statistical significance for several specific comparisons. We therefore included all data from both communities in our analyses.

Scientificethics:TheKEMRIEthical Review Committee and the CDC Institutional Review Board approved the protocol for the present research. Parents or guardians of all subjects provided informed consent.

\section{RESULTS}

Specimen and population characteristics: A total of 1303 urban patients and 1247 rural patients were enrolled during the respective periods of surveillance. The mean age of urban enrollees was 12 months compared with 16 months among rural enrollees $(\mathrm{P}<0.001$, Table 1). In addition, the proportional distribution of age group membership was skewed for both communities, with less than $5 \%$ of children being older than 48 months (Figure 1). Urban patients presented to study clinics for evaluation on average one day earlier after onset of symptoms than rural patients (mean 3.6 days vs. 4.4 days, IQR 2-4 days vs. $2-5$ days, $\mathrm{P}<0.001)$. Although the frequency of nausea (61\% urban vs. $64 \%$ rural) and vomiting (60\% vs. $62 \%$, respectively) accompanying the diarrhoeal illness werenot significantly different between communities, urban patients reported significantly less subjective fever $(73 \%$ vs. $82 \%$, OR $0.59,95 \%$ CI $0.48-0.71, \mathrm{P}<0.001$ ) and abdominal cramping ( $63 \%$ vs. $75 \%$, OR $0.53,95 \%$ $\mathrm{CI} 0.45-0.64, \mathrm{P}<0.001)$. Asignificantly greater fraction of urban patients presented with watery diarrhoea (95\% vs. $71 \%$, OR $6.42,95 \%$ CI $4.59-8.98, \mathrm{P}<0.001$ ) whereas urban children were less likely to present with bloody(3\%vs. 10\%, OR 0.35, 95\% CI 0.25-0.51, $\mathrm{P}<0.001)$ ormucoid $(1 \%$ vs. $14 \%$, OR $0.28,95 \% \mathrm{CI}$ 0.22-0.37, $\mathrm{P}<0.001)$ diarrhoea.

\section{Figure 1}

Age distribution of patients presenting with diarrhoea to urban and rural clinics, Western Kenya, May 1997-

October 2003

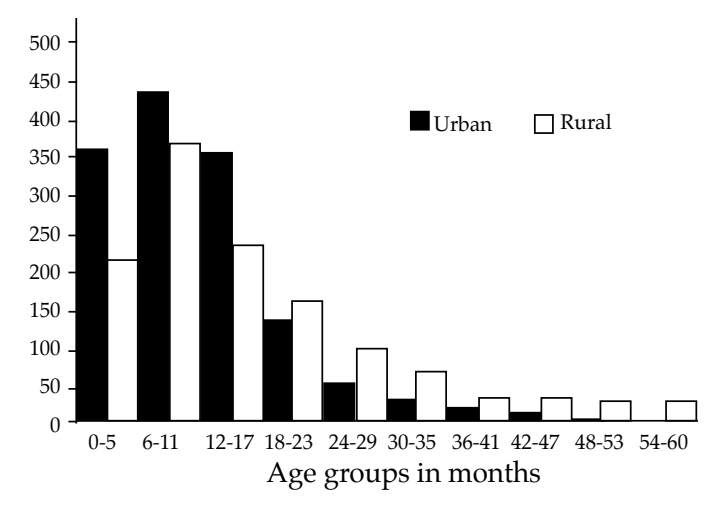




\section{Table 1}

Comparison of demographics, clinical characteristics, and bacterial enteric pathogens isolated from urban and rural paediatric outpatients with diarrhoea: Western Kenya, May 1997-October 2003a

\begin{tabular}{|c|c|c|c|c|}
\hline & Urban $(n=1303)$ & Rural $(\mathrm{n}=1247)$ & OR $(95 \% \mathrm{CI})^{\mathrm{b}}$ & P-value \\
\hline \multicolumn{5}{|l|}{ Demographics } \\
\hline Mean age, months & $12(5-16)$ & $16(7-22)$ & -- & $<0.001$ \\
\hline \multicolumn{5}{|l|}{ Interquartile range [IQR] } \\
\hline Female & $592 / 1,300(46 \%)$ & $588 / 1,245(47 \%)$ & $1.05(0.89-1.23)$ & 0.56 \\
\hline \multicolumn{5}{|l|}{ Clinical data } \\
\hline $\begin{array}{l}\text { Mean days from onset } \\
\text { to clinic visit (IQR) }\end{array}$ & $3.6(2-4)$ & $4.4(2-5)$ & -- & $<0.001$ \\
\hline Subjective fever & $950 / 1,300(73 \%)$ & $1020 / 1,244(82 \%)$ & $0.59(0.48-0.71)$ & $<0.001$ \\
\hline Abdominal cramping & $811 / 1,296(63 \%)$ & $886 / 1,184(75 \%)$ & $0.53(0.45-0.64)$ & $<0.001$ \\
\hline Nausea & $799 / 1,300(61 \%)$ & $764 / 1,191(64 \%)$ & $0.87(0.73-1.02)$ & 0.089 \\
\hline Vomiting & $784 / 1,300(60 \%)$ & $775 / 1,245(62 \%)$ & $0.88(0.74-1.04)$ & 0.13 \\
\hline \multicolumn{5}{|l|}{ Quality of diarrhoea } \\
\hline Watery ${ }^{c}$ & $1,240(95 \%)$ & $904(71 \%)$ & $6.42(4.59-8.98)$ & $<0.001$ \\
\hline Bloody ${ }^{\mathrm{d}}$ & $45(3 \%)$ & $124(10 \%)$ & $0.35(0.25-0.51)$ & $<0.001$ \\
\hline Mucoide $^{\mathrm{e}}$ & $7(1 \%)$ & $182(14 \%)$ & $0.28(0.22-0.37)$ & $<0.001$ \\
\hline Uncharacterised $^{\mathrm{f}}$ & $11(1 \%)$ & $55(4 \%)$ & $0.20(0.10-0.38)$ & $<0.001$ \\
\hline \multicolumn{5}{|l|}{ Stool culture results } \\
\hline No growth & $3(<1 \%)$ & $8(1 \%)$ & $0.41(0.11-1.62)$ & 0.21 \\
\hline Normal growth & $991(76 \%)$ & $937(75 \%)$ & $1.05(0.88-1.27)$ & 0.58 \\
\hline Yielded a pathogen & $309(24 \%)$ & $302(25 \%)$ & $0.97(0.80-1.16)$ & 0.72 \\
\hline Yielded $>{ }^{1}$ pathogen & $16(1 \%)$ & $19(2 \%)$ & $0.85(0.43-1.69)$ & 0.65 \\
\hline Total pathogens isolated & 325 & 321 & & \\
\hline \multicolumn{5}{|l|}{ Pathogen isolation rates ${ }^{\mathrm{g}}$} \\
\hline Bloody & $14 / 45(31 \%)$ & $47 / 124(38 \%)$ & $1.01(0.84-1.22)$ & 0.93 \\
\hline Watery $^{\mathrm{d}}$ & $290 / 1,240(23 \%)$ & $198 / 886(24 \%)$ & $1.05(0.87-1.28)$ & 0.60 \\
\hline Mucoide $^{e}$ & $2 / 7(29 \%)$ & $47 / 182(26 \%)$ & $0.98(0.81-1.19)$ & 0.85 \\
\hline Uncharacterised $^{\mathrm{f}}$ & $3 / 11(27 \%)$ & $10 / 55(18 \%)$ & $0.96(0.80-1.16)$ & 0.69 \\
\hline \multicolumn{5}{|l|}{ Species isolated } \\
\hline Campylobacter & $224 / 1,303(17 \%)$ & $193 / 1,247(15 \%)$ & $1.04(0.82-1.29)$ & 0.70 \\
\hline All Shigella species & $51 / 1,303(4 \%)$ & $65 / 1,247(5 \%)$ & $0.98(0.66-1.44)$ & 0.90 \\
\hline S. flexneri & $33 / 51(65 \%)$ & $38 / 65(58 \%)$ & $1.34(0.62-2.91)$ & 0.46 \\
\hline S. sonnei & $7 / 51(14 \%)$ & $16 / 65(25 \%)$ & $0.48(0.19-1.19)$ & 0.11 \\
\hline S. boydii & $4 / 51(8 \%)$ & $5 / 65(8 \%)$ & $0.95(0.23-3.87)$ & 0.95 \\
\hline S. dysenteriae, non-type 1 & $3 / 51(6 \%)$ & $3 / 65(5 \%)$ & $1.65(0.29-9.33)$ & 0.57 \\
\hline S. dysenteriae, type 1 & $0 / 51(0 \%)$ & $3 / 65(5 \%)$ & $N A^{h}$ & NA \\
\hline S.spp. unserotyped & $4 / 51(8 \%)$ & $0 / 65(0 \%)$ & NA & NA \\
\hline Nontyphoidal Salmonella & $49 / 1,303(4 \%)$ & $61 / 1,247(5 \%)$ & $0.72(0.48-1.06)$ & 0.10 \\
\hline Group B Salmonella & $31 / 49(43 \%)$ & $24 / 61(39 \%)$ & $2.91(1.32-6.46)$ & 0.008 \\
\hline Group C1 Salmonella & $2 / 49(4 \%)$ & $2 / 61(3 \%)$ & $1.56(0.21-11.6)$ & 0.67 \\
\hline Group C2 Salmonella & $3 / 49(6 \%)$ & $5 / 61(8 \%)$ & $0.69(0.16-3.01)$ & 0.63 \\
\hline Group D Salmonella & $10 / 49(20 \%)$ & $19 / 61(31 \%)$ & $0.57(0.23-1.37)$ & 0.21 \\
\hline Group E Salmonella & $0 / 49(0 \%)$ & $5 / 61(8 \%)$ & NA & NA \\
\hline Group F Salmonella & $0 / 49(0 \%)$ & $3 / 61(5 \%)$ & NA & NA \\
\hline Salmonella, other ${ }^{\mathrm{i}}$ & $1 / 49(2 \%)$ & $2 / 61(3 \%)$ & NA & NA \\
\hline Salmonella spp., not grouped & $2 / 49(4 \%)$ & $1 / 61(2 \%)$ & $2.1(0.19-25.47)$ & 0.53 \\
\hline Salmonella enterica serotype Typh & ii $1 / 1,303(1 \%)$ & $0 / 1,247(0 \%)$ & NA & NA \\
\hline Vibrio cholerae $\mathrm{O} 1$ & $0 / 1,303(0 \%)$ & $1 / 1,247(<1 \%)$ & NA & NA \\
\hline Other vibrio spp. & $0 / 1,303(0 \%)$ & $1 / 1,247<1 \%)$ & NA & NA \\
\hline
\end{tabular}


a. Surveillance data for Kisumu (urban) is limited to October 2001-october 2003. Asembo Bay(rural) is limited to May 1997-June 2003 and is a subset of a previously published data consisting of only patients 0-5 years old. ${ }^{6}$

b. $\quad$ Adjusted by age.

c. Reported "watery diarrhoea" but denied "bloody diarrhoea", excluded unknown responses for "bloody diarrhoea" d. Reported "bloody diarrhoea" but may also have reported "watery" and/ or "mucoid diarrhoea."

e. Reported "mucoid diarrhoea" alone. denied both "bloody" and "watery diarrhoea", excluded unknowns responses for "bloody diarrhoea" and for "watery diarrhoea.'

f. Reported "uncharacterised" all responses regarding stool quality were blank

g. $\quad$ For this table pathogens include: Shigella Salmonella, Vibrio, and Campylobacter.

h. NA Not Applicable: Zero value or collinearity prevents calculating adjusted OR and P-value;

i. In Kisumu there was one isolate of Salmonella II 1, 13, 23:29 that could not be serogrouped; in Asembo Bay there was one each of, Salmonella enterica serotype Leoben (Group M), Salmonella enterica serotype Taiping (Group G)

Bacterial isolates: Bacterial isolation rates were comparablebetween theurban and rural communities: $24 \%$ vs. $25 \%$ of specimens, respectively, yielded at least one bacterial pathogen and $1 \%$ vs. $2 \%$ of specimens yielded $>1$ pathogen (Table 1). There were no significant differences in isolation rates according to type of diarrhoea for each community: $31 \%$ vs. $38 \%$ for bloody diarrhoea, $29 \%$ vs. $26 \%$ for watery diarrhoea, and $23 \%$ vs. $24 \%$ for mucoid diarrhoea. Campylobacter was the most common bacterial pathogen identified; isolated from $17 \%$ (urban) vs. 15\% (rural) of all specimens. Whether considered together or individually, there was no significant difference in the rates of isolation of Shigella serogroups from the specimens of patients from urban compared to rural communities (4\% vs. $5 \%$ of all specimens). Among the Shigella isolates, the proportion of each species in urban vs. rural sites was $65 \%$ vs. $58 \%$ for S.flexneri, $14 \%$ vs. $25 \%$ for $S$. sonnei, $8 \%$ vs. $8 \%$ for S. boydii, $6 \%$ vs. $5 \%$ for non-type $1 S$. dysenteriae, $0 \%$ vs. $5 \%$ for type $1 S$. dysenteriae; $8 \%$ vs. $0 \%$ were not serogrouped(Table 1$)$. S.flexneri serotypes included $2 \mathrm{a}(21 \%$ vs. $24 \%), 1 \mathrm{~b}(15 \%$ vs. $24 \%)$, $3 \mathrm{a}(15 \%$ vs. $13 \%), 4 \mathrm{a}(9 \%$ vs. $0 \%)$ and $6(6 \%$ vs. $18 \%)$ (Table 2). However, the sub-analysis of serogroups and serotypes of Salmonella species revealed that Group B Salmonella and Salmonella enterica serotype Typhimurium, which belongs to Group B, were more common in urban patients (43\% vs. 39\%, OR 2.91, 95\% CI 1.32-6.46, P0.008 and 31\% vs. 18\%, OR 3.40, $95 \%$ CI 1.29-8.96, $\mathrm{P}=0.014$, respectively). The other most common nontyphoidal Salmonella isolated were Salmonella enterica serotype Enteritidis ( $21 \%$ vs. $18 \%)$, Salmonella enterica serotype Newport (6\% vs. 2\%), and Salmonella enterica serotype Heidelberg (10\% vs. $16 \%$ ) (Table 2). One specimen from an urban patient yielded Salmonella enterica serotype Typhi.

Table 2

Shigella and nontyphoidal Salmonella serotypes from children with diarrhoea: Western Kenya, May 1997-October $2003^{a}$

\begin{tabular}{|c|c|c|c|c|}
\hline & Urban & Rural & OR $(95 \% \mathrm{CI})^{\mathrm{b}}$ & $P$-value \\
\hline \multicolumn{5}{|l|}{ S. flexneri serotypes } \\
\hline $2 a$ & $7 / 33(21 \%)$ & $9 / 38(24 \%)$ & $0.78(0.29-2.13)$ & 0.63 \\
\hline $1 b$ & $5 / 33(15 \%)$ & $9 / 38(24 \%)$ & $0.76(0.23-2.51)$ & 0.66 \\
\hline $3 a$ & $5 / 33(15 \%)$ & $5 / 38(13 \%)$ & $1.31(0.35-4.92)$ & 0.69 \\
\hline $4 a$ & $3 / 33(9 \%)$ & $0 / 38(0 \%)$ & $\mathrm{NA}^{\mathrm{c}}$ & NA \\
\hline 6 & $2 / 33(6 \%)$ & $7 / 38(18 \%)$ & $0.28(0.05-1.46)$ & 0.13 \\
\hline Other $^{\mathrm{d}}$ & $1 / 33(3 \%)$ & $3 / 38(8 \%)$ & $0.39(0.04-4.03)$ & 0.43 \\
\hline Unserotyped & $10 / 33(30 \%)$ & $5 / 38(13 \%)$ & $3.3(0.94-11.7)$ & 0.063 \\
\hline \multicolumn{5}{|l|}{ Nontyphoidal Salmonella serotypes } \\
\hline Salmonella enterica serotype Typhimurium (Group & B) $15 / 49(31 \%)$ & $11 / 61(18 \%)$ & $3.40(1.29-8.96)^{\mathrm{d}}$ & 0.014 \\
\hline Salmonella enterica serotype Enteritidis (Group D) & $7 / 49(21 \%)$ & $18 / 61(30 \%)$ & $0.62(0.22-1.71)^{\mathrm{d}}$ & 0.35 \\
\hline Salmonella enterica serotype Heidelberg (Group B) & $5 / 49(10 \%)$ & $10 / 61(16 \%)$ & $0.96(0.29-3.23)^{\mathrm{d}}$ & 0.95 \\
\hline Salmonella enterica serotype Newport (Group C2) & $3 / 49(6 \%)$ & $1 / 61(2 \%)$ & $5.05(0.50-51.0)^{\mathrm{d}}$ & 0.17 \\
\hline Salmonella enterica serotype Kenya (Group C1) & $2 / 49(4 \%)$ & $0 / 61(0 \%)$ & NA & NA \\
\hline $\begin{array}{l}\text { Salmonella enterica serotype 4,S,12:A:-Monophasic } \\
\text { (Group B) }\end{array}$ & $2 / 49(4 \%)$ & $0 / 61(0 \%)$ & NA & NA \\
\hline Salmonella enterica serotype Aberdeen (Group F) & $0 / 49(0 \%)$ & $3 / 61(5 \%)$ & NA & NA \\
\hline Salmonella enterica serotype Uganda (Group E) & $0 / 49(0 \%)$ & $3 / 61(5 \%)$ & NA & NA \\
\hline
\end{tabular}




\begin{tabular}{lcccc} 
Salmonella enterica serotype Chailey (Group C2) & $0 / 49(0 \%)$ & $2 / 61(3 \%)$ & NA & NA \\
Other & $1 / 49(2 \%)$ & $9 / 61(15 \%)$ & NA & NA \\
Unserotyped & $14 / 49(29 \%)$ & $4 / 61(7 \%)$ & $5.87(1.76-19.6)$ & 0.004 \\
\hline
\end{tabular}

a. Surveillance data for Kisumu (urban) is limited to October 2001-october 2003. Asembo Bay (rural) is limited to May 1997-June 2003 and is a subset of a previously published data consisting of only patients 0-5 years old (6).

b. $\quad$ Adjusted by age.

c. $\quad \mathrm{NA}=$ Not Applicable: Zero value prevents or collinearity prevents calculating adjusted OR and P-value.

d. In Kisumu there was one isolate each Shigella flexneri serotype $\mathrm{X}$ variant. In Asembo Bay there was one isolate each of Shigella flexneri serotype 1 A, Shigella flexneri serotype 3B, and Shigella flexneri serotype 4B.

e. $\quad$ Adjusted by age and unserotyped Salmonella

f. In Kisumu there was one isolate of Salmonella II 1, 13, 23:229. In Asembo Bay there was one isolate each of Salmonella enterica serotype Bardo (Group C2), Salmonella enterica serotype turi (Group B), Salmonella enterica serotype Leoben (Group M), Salmonella enterica serotype Lindenburg (Group Cl), Salmonella enterica serotype 16 , 7,[I4]:b:- (Group C1), Salmonella enterica serotype Taiping (Group G), Salmonella enterica serotype Zanzibar (Group $\mathrm{EI})$, and one nontypable (Group C2) and one nontypable (not serogrouped).

Table 3

Antimicrobials taken by paediatric outpatients with diarrhoea before seeking medical attention, Western Kenya, May 1997-October 2003 ${ }^{a}$

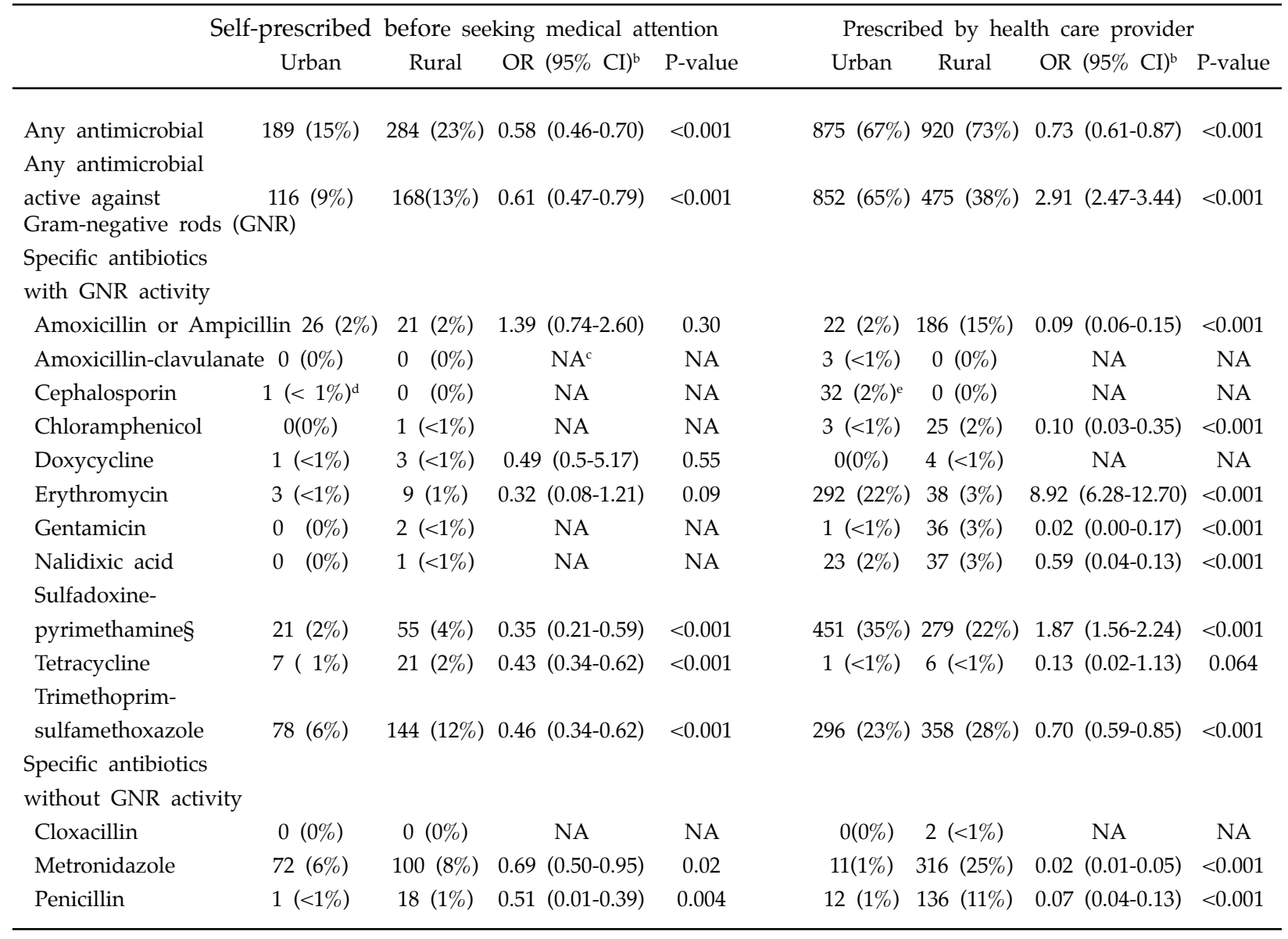

a. Surveillance data for Kisumu (urban) is limited to October 2001 - October 2003. Asembo Bay (rural) is limited to May 1997-June 2003 and is a subset of a previously published data consisting of only patients 0-5 years old; (6)

b. Adjusted by age; d Cephalexin (1); e Includes cephalexin (6), cefadroxil (12), cefazolin (3), cefaclor (10), and cefufur (I); ‘11 Sulfonamide-containing anti malarial.

c. $\quad \mathrm{NA}=$ Not applicable: Zero value prevents or collinearity prevents calculating adjusted OR and P-value

d. Cephalexin (1)

e. Includes cephalexin (6)

f. Sulfonamide - Containing 
Although age-specific isolation rates varied significantly by pathogen, patterns of isolation were similar in urban and rural populations (Figure 2). For instance, an analysis of Campylobacter isolation rates among infants using one month intervals indicated that the peak isolation rate of 31 per 100 diarrhoea cases occurred among urban infants who were 12 months old, while a peak isolation rate of 29 per 100 diarrhoea cases occurred among rural children who were 10 months old. Shigella isolation rates tended to increase with increasing age in urban and rural patients ( $\beta$ 0.04, 95\% CI 0.02-0.05, $\mathrm{P}<0.001 ; \beta 0.03$, $95 \% \mathrm{CI} 0.01-0.04, \mathrm{P}<0.001)$. The age-specific isolation rates for nontyphoidal Salmonella remained stable across age categories $(\beta-0.002,95 \% \mathrm{CI}-0.01-0.02, \mathrm{P}$ $0.74 ; \beta-0.006,95 \%$ CI $-0.02-0.005$, P 0.24$)$.

Associations with presenting signs and symptoms: Shigela was significantly more likely to be isolated from bloody specimens in both communities (OR 7.17,95\% CI 2.84-16.39, $\mathrm{P}<0.001$ vs. OR 7.35,95\% CI 4.10-12.96, $\mathrm{P}<0.001)$. Isolation of Campylobacter or nontyphoidal Salmonella was not associated with any type of diarrhoea eg watery, blody, etc. There was no other association among isolates with other presenting symptoms (such as, fever, abdominal cramps, nausea, vomiting) by community.

Diarrhoeal treatment, antimicrobial use and susceptibility testing: Before presenting to the clinic, urban enrollees were less likely to have taken antimicrobials $(15 \%$ vs. $21 \%$, OR $0.58,95 \%$ CI $0.46-0.70, \mathrm{P}<0.001)$ or herbal remedies ( $3 \%$ vs. $16 \%$, OR $0.17,95 \%$ CI $0.12-0.23$, $\mathrm{P}<0.001)$. Use of ORS before the visit was equally low in both settings ( $4 \%$ vs. $4 \%$ ).

The World Health Organisation recommends antimicrobial therapy only for treatment for dysentery and for moderate to severe cholera with an antibiotic active against GNR in additional to standard hydration therapy (10). Antibiotics active against GNR include TMP/SMX, aminopenicillins, third-generation cephalosporins, nalidixic acid, and fluoroquinolones. Patients were less likely to be prescribed any antimicrobial agent in the urban community ( $67 \%$ vs. $73 \%$, OR $0.73,95 \%$ CI $0.61-0.87, \mathrm{P}$ $<0.001$ ) and were also less likely to be prescribed more than one agent ( $21 \%$ vs. $33 \%$, OR $0.68,95 \%$ CI $0.62-$ $0.75, \mathrm{P}<0.001)$. Antimicrobials prescribed at the urban clinic were more likely to have activity against GNR (65\% vs. $38 \%$, OR $2.91,95 \%$ CI 2.47-3.44, P <0.001). In terms of specific antimicrobial agents, providers at the urban clinic were significantly more likely to prescribe erythromycin (22\% vs. $3 \%$, OR $8.92,95 \%$ CI6.28-12.70, $\mathrm{P}<0.001$ ) and significantly less likely to prescribe aminopenicillins ( $2 \%$ vs. $15 \%$, OR $0.09,95 \%$ CI $0.06-0.15, \mathrm{P}<0.001)$ or metronidazole ( $1 \%$ vs. $25 \%$, OR $0.02,95 \%$ CI $0.01-0.05, \mathrm{P}<0.001)$. Our regression model examining the differences in treatment of dysentery revealed that despite the fact that bloody diarrhoea was less commoninurban patients, children presenting with bloody diarrhoea to the urban clinic were more likely to have been prescribed ORS (OR $11.63,95 \%$ CI1.07-126.3, P 0.04) and an antibiotic with GNR activity (OR 4.24,95\% CI 1.99-9.04, $\mathrm{P}<0.001$ ).

Antimicrobial susceptibility patterns were also similar for isolates from urban and rural patients (Table4).Susceptibilities of Shigella and nontyphoidal Salmonella to TMP-SMZ were low (17\% urban vs. $25 \%$ rural) (Table 4). The only antimicrobials to which more than $80 \%$ of Salmonella and Shigella isolates were susceptible were ciprofloxacin, ceftriaxone, nalidixic acid, kanamyicin, and gentamicin. Susceptibilities of Campylobacter to the antibiotics tested were $>80 \%$ in both communities except for clindamycin, erythromycin, TMP-SMZ and nalidixic acid (Table 4 ). Only $33 \%$ to $45 \%$ of isolates were susceptible to erythromycin, but more than half of isolates demonstrated intermediate susceptibility. Overall, providers at the urban clinic were significantly less likely to prescribe antibiotics to which the patient's isolate was resistant (19\% vs. $81 \%$, OR $0.22,95 \%$ CI $0.12-0.45, \mathrm{P}<0.001)$. 
Figure 2

Comparison of age-specific isolation rates for Campylobacter, Shigella, and Salmonella from patients ages 0-5 years by location of residence, Western Kenya, May 1997-October 2003.
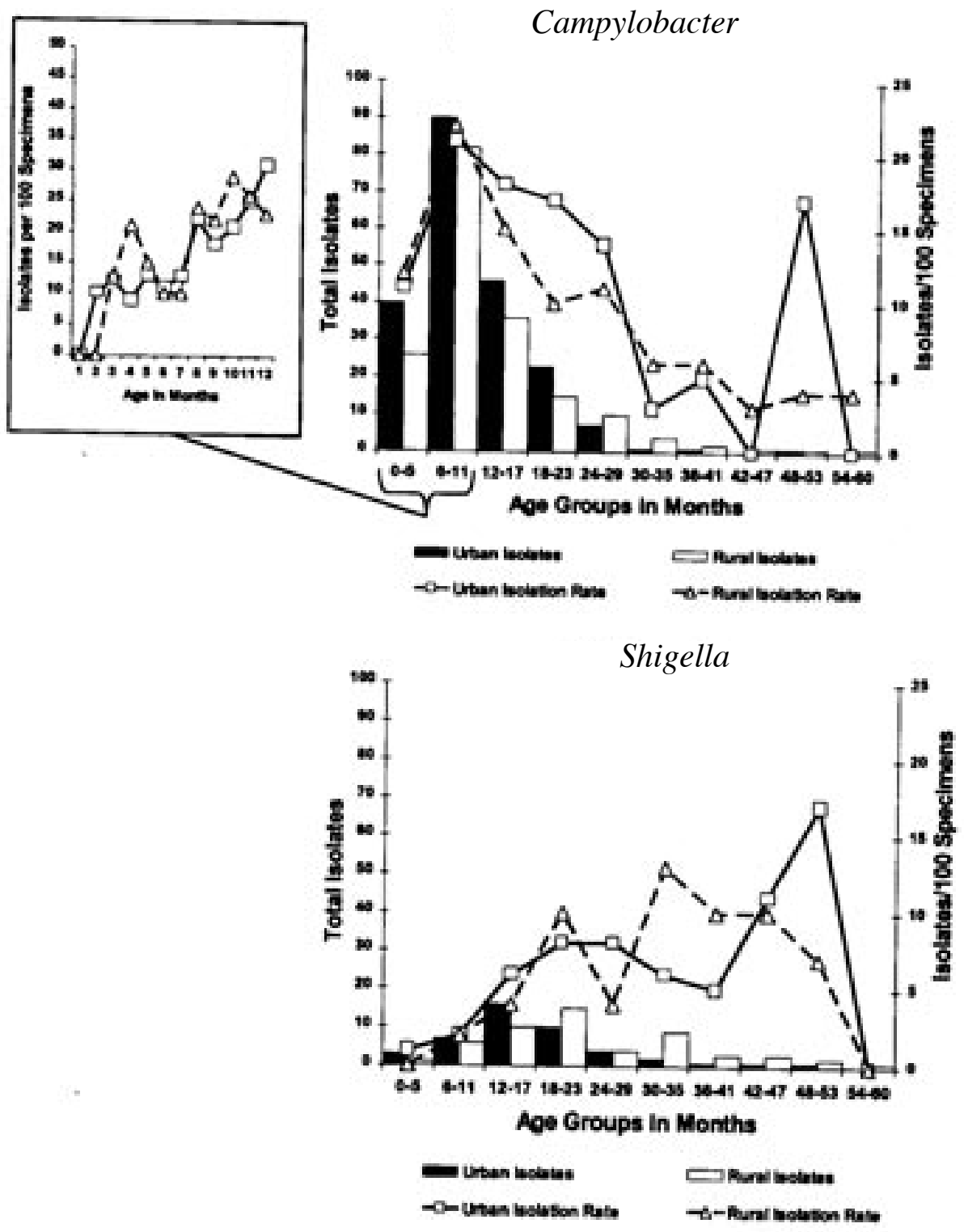

Nontyphoidal Salmonella

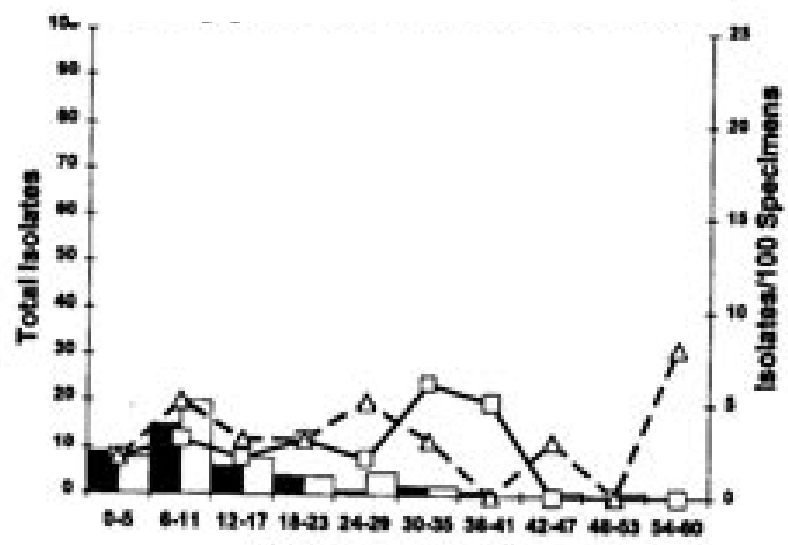

Aoe Groups in Months

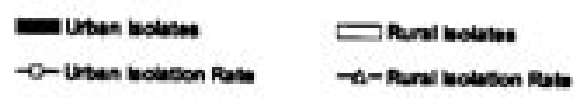


Table 4

Antimicrobial susceptibility patterns among selected Shigella serogroups and Salmonella serotypes isolated from children with diarrhoea: Western Kenya, May 1997-October 2003. ${ }^{a}$

\begin{tabular}{|c|c|c|c|c|c|c|c|c|c|c|c|c|c|c|c|}
\hline \multirow[b]{2}{*}{ Pathogen (Number) } & \multicolumn{15}{|c|}{ Percent susceptible isolates ${ }^{b}$} \\
\hline & $\mathrm{CH}$ & TS & $\mathrm{TE}$ & $\mathrm{CP}$ & NA & A & SZ & $S$ & $\mathrm{~K}$ & G & $\mathrm{CT}$ & $\mathrm{A}-\mathrm{C}$ & CL & $\mathrm{E}$ & CES \\
\hline \multicolumn{16}{|l|}{ Urban } \\
\hline \multicolumn{16}{|l|}{ Shigella $\mathcal{E}$ non-typhoidal } \\
\hline Salmonella $(n=99) \mathrm{T}$ & 57 & 17 & 19 & 100 & 93 & 31 & 7 & 16 & 84 & 84 & 100 & 38 & - & - & - \\
\hline Shigella $(\mathrm{n}=51)$ & 44 & 2 & 9 & 100 & 98 & 27 & 6 & 8 & 100 & 98 & 100 & 37 & - & - & - \\
\hline S. flexneri $(\mathrm{n}=33)$ & 24 & 3 & 3 & 100 & 100 & 18 & 9 & 9 & 100 & 97 & 100 & 27 & - & - & - \\
\hline S. sonnei $(\mathrm{n}=7)$ & 100 & 0 & 0 & 100 & 100 & 86 & 0 & 0 & 100 & 100 & 100 & 100 & - & - & - \\
\hline Nontyphoidal Salmonella $(\mathrm{n}=48)$ & 72 & 33 & 31 & 100 & 87 & 35 & 8 & 25 & 42 & 69 & 100 & 40 & - & - & - \\
\hline S. ser. Typhimurium $(\mathrm{n}=15)$ & 93 & 20 & 33 & 100 & 100 & 7 & 0 & 13 & 27 & 27 & 100 & 20 & - & - & - \\
\hline S. ser. Enteritidis $(\mathrm{n}=7)$ & 40 & 40 & 0 & 100 & 40 & 29 & 14 & 29 & 86 & 100 & 100 & 29 & - & - & - \\
\hline $\begin{array}{l}\text { Campylobacter }(n=24) \\
\text { Rural }\end{array}$ & 92 & 88 & 92 & 92 & 92 & - & - & - & - & - & - & - & 79 & 33 & 92 \\
\hline $\begin{array}{l}\text { Shigella } \mathcal{E} \text { nontyphoidal } \\
\text { Salmonella }(n=122)\end{array}$ & 52 & 25 & 21 & 100 & 94 & 44 & 12 & 16 & 93 & 93 & 100 & 48 & - & - & - \\
\hline Shigella $(n=65)$ & 43 & 3 & 3 & 100 & 97 & 35 & 3 & 2 & 100 & 100 & 100 & 42 & - & - & - \\
\hline S. flexneri $(\mathrm{n}=38)$ & 6 & 6 & 0 & 100 & 94 & 13 & 5 & 3 & 100 & 100 & 100 & 21 & - & - & - \\
\hline S. sonnei $(\mathrm{n}=16)$ & 100 & 0 & 0 & 100 & 100 & 94 & 0 & 0 & 100 & 100 & 100 & 94 & - & - & - \\
\hline Nontyphoidal Salmonella $(\mathrm{n}=57)$ & 63 & 51 & 43 & 100 & 92 & 54 & 23 & 32 & 84 & 84 & 100 & 56 & - & - & - \\
\hline S. ser. Typhimurium $(n=10)$ & 100 & 25 & 63 & 100 & 75 & 20 & 10 & 10 & 20 & 20 & 100 & 20 & - & - & - \\
\hline S. ser. Enteritidis $(\mathrm{n}=17)$ & 6 & 6 & 6 & 100 & 100 & 12 & 12 & 6 & 100 & 100 & 100 & 18 & - & - & - \\
\hline Campylobacter $(n=80)$ & 94 & 73 & 83 & 95 & 75 & - & - & - & - & - & - & - & 71 & 49 & 93 \\
\hline
\end{tabular}

a. Surveillance data for Kisumu (urban) is limited to October 2001-October 2003. Asembo Bay (rural) is limited to May 1991-June 2003 and is a subset of a previously published data consisting of only patients 0-5 years old (6).

b. $\quad \mathrm{CH}=$ chloramphenicol; $\mathrm{TS}=$ trimethoprim-sulfamethoxazole; $\mathrm{T}=$ tetracycline; $\mathrm{CP}=$ ciprofloxacin; $\mathrm{NA}=$ nalidixic acid; $\mathrm{A}=$ ampicillin; $\mathrm{SZ}=$ sulfisoxazole; $\mathrm{S}=$ streptomycin; $\mathrm{K}=$ kanamycin; $\mathrm{G}$ = gentamicin; $\mathrm{CT}=$ ceftriaxone; $\mathrm{A}-\mathrm{C}$ = amoxicillin-clavulanic acid, $\mathrm{CL}=$ clindamycin; $\mathrm{E}=$ erythromycin; $\mathrm{CE}=$ cephalothin.

\section{DISCUSSION}

In these urban and rural communities in western Kenya, we found several similarities in the characteristics of diarrhoeal diseases in children $\leq 5$ years old. Campylobacter was the predominant bacterial pathogen isolated from the stools of children with diarrhoea overall, with a peak around the first year of life in both communities. This finding is consistent with previous studies of Campylobacter in Africa and Asia (11-13). However, isolation of Campylobacter spp. from children without diarrhoea is also common in developing countries (14). In some studies, no statistically significant difference was noted in Campylobacter isolation rates in certain age groups (15).

Simango and Nyahanan (16) examined rates of Campylobacter infection specifically among urban patients with diarrhoea and reported that urban patients tended to be older; however the conclusion was based on only 27 Campylobacter-positive patients ages 0 to 5 years. In another study comparing bacterial aetiologies of diarrhoea in urban vs. rural settings by Obi et al (2), Campylobacter was reported to be more common in patients with diarrhoea who presented to an urban clinic; however, patients were 1 to 45 years old and age-specific isolation rates were not compared or reported. In contrast, we found that urban patients tended to be younger and presented for care sooner after illness onset. This difference may reflect better access to healthcare in the urban setting $(17,18)$. Although dysentery was more common among rural patients, there were no significant differences in the isolation of specific bacterial pathogen associated with dysentery (Shigella). One explanation could be that a parasitic cause of dysentery, such as Entamoeba histolytica, was more common in the rural setting $(19,20)$. However, testing for parasitic agents was not routinely conducted for specimens in this study. 
Chickens are a common source of Campylobacter and children may develop protective antibodies against infection in a number of different ways other than through enteric infection. Campylobacter was isolated from $77 \%$ of retail poultry meat sold in Nairobi, Kenya (21). Oberhelman et al. (22) found that isolation of the same Campylobacter strain from a household healthy chicken and a healthy human within one month was highly protective against diarrhoea. Coke et al. (23) hypothesized that maternal antibodies transferred transplacentally and through breast milk may provide partial protection from Campylobacter infection in infants less than one year of age. This protection wanes as a function of half-life of maternal antibodies.

The identification of Salmonella Typhimurium and Salmonella Enteritidis as the most common nontyphoidal Salmonella has been noted in previous studies of children with bacteremia or diarrhoea (24, 25) conducted in referral hospitals in Nairobiand Kilifi, Kenya. However, in both of these studies Salmonella Enteritidis was the most common serotype. In the present study, Salmonella Typhimurium was isolated significantly more often than Salmonella Enteritidis in the urban community; in contrast, Salmonella Typhimurium predominated among nontyphoidal Salmonella in the rural community. In contrast to the bacteremia study in Kilifi, the predominance of Salmonella Typhimurium over Salmonella Enteritidis in our study of gastroenteritis may reflect a difference in serotype specific invasiveness, or perhaps environmental, cultural, or even genetic differences in these two populations. The finding of Salmonella Heidelberg as a common cause of nontyphoidal paediatric salmonellosis has, to our knowledge, not previously been reported in Africa.

Where differences between urban and rural communities were most evident was in how patients and theirhealth care providers responded to theneed for treatment. Rural patients were morelikely tohave taken antimicrobials or herbal remedies before presenting to a clinic. Antimicrobials are readily accessible to patients without a prescription in Kenya, and parents of children in rural communities may treat their children more often than parents in urban areas due to differences in access to care (for instance, access to care is less readily available to rural patients therefore parent self-treat us an alternative). Although greater self-treatment might be hypothesized to result in differences in bacterial pathogens' antibiotic susceptibility, we found antibiotic sensitivity profiles between urban and rural bacterial isolates were similar.

Significant differences were found in treatment practices among providers at the urban and rural clinics. The lessfrequent prescription of antimicrobials, the more frequent choice of antimicrobials with activity against GNR, and the more frequent use of hydration (ORS and IVF) suggest the providers at the urban clinic were more knowledgeable of and / or more compliant with WHO recommendations for the treatment of diarrhoea (10).

According to published reports, between 5$38 \%$ of children with malaria may present with diarrhoea as a symptom $(26,27)$; therefore, diarrhoea should not be considered a localizing symptom. WHO recommends empiric treatment for malaria in the case of children with non-localizing fever (10). Although subsequent studies have questioned the association between malaria and diarrhoea $(28,29)$, use of sulfadoxine-pyrimethamine (SP, which was the first-line antimalarial treatment at the time of the study) was still significantly more common among urban patients, suggesting urban providers were more compliant with WHO recommendations (10) than their rural peers.

The greater empiric use of erythromycin, an appropriate choice in the treatment of Campylobacter enteritis if susceptible, in the urban clinic suggests these providers were making a more informed choice, possibly due to an awareness of the high incidence of Campylobacter infection in infants and young children. In the rural clinics, the high rates of metronidazole prescription for diarrhoea suggest less informed empiric treatment decision making; metronidazole is effective for amoebiasis and giardiasis but these infections rarely cause diarrhoea in children in this region (CDC unpublished data) (28).

Our analysis is limited to illnesses among children receiving care at clinics where we conducted surveillance; we, therefore, cannot providepopulationbased incidence data. However, since our sampling methods were systematic we believe our isolation rates are representative of the disease incidence in the area where the study was conducted. Our study was also limited by inclusion of surveillance data from non-overlapping time periods, which could introduce period effects. However, separate analysis limited to overlapping time periods revealed no difference in trends; only a loss of power due to low sample size in the rural communities. Moreover, previous analysis of rural data, of which of our study used a subset, showed no major time-related changes that would alter the causes of diarrhoeal illness, likelihood of presentation, or health care provider treatment practices between non-overlapping periods (6).

In summary, although most characteristics of diarrhoeal disease and its bacterial aetiology were similar in paediatric patients presenting to urban and rural clinics, we documented important differences in health-seeking behaviour and provider treatment practices. Rural patients were more likely to self-treat with antibiotics or herbal remedies 
before seeking medical attention. Treatment by urban providers was more consistent with WHO recommendations. However, due to the decreased antimicrobial susceptibilities of the most frequently isolated diarrhoeal bacterial pathogens among both urban and rural children, the efficacy of the empiric antibiotic therapy provided was likely limited.

\section{ACKNOWLEDGEMENTS}

To P. Phillips-Howard, N. Puhr, and P. Heaton from CDC and J. Achieng, C. Ayuyo, H. Moige, J. Juma, H. Joseph, G. Ochola, C. Okonji, M. Onyango, J. Okulo, S. Odhiambo, L.Okuogo,J.Okulo,M.Ombak, N.Omondi, J. Orurre, J. Otieno, J. Oyiengo, E. Shoute, R. Wanga, B. Watanga, and W. Y. Ongo, (Kenya Medical Research Institute, Kisumu, Kenya) for helping with thisstudy. We also thank the Director of the Kenya Medical Research Institute for permission to publish this paper and the residents of Kisumu and of Asembo Bay, Kenya.

Ethical Approval: The authors have complied with the journals policy on ethical standards. Animal research was not part of this study.

\section{REFERENCES}

1. Parashar, U.N., Bresee, J.S. and Glass, R. I. The global burden of diarrhoeal disease in children. Bull. World Hlth Org. 2003; 81:4:236.

2. Obi, C. L., Coker, A.O., Epoke, J. and Ndip, R.N. Enteric bacterial pathogens in stools of residents of urban and rural regions in Nigeria: a comparison of patients with and without diarrhoea and controls without diarrhoea. J. Diarr. Res. 1997; 15: 4:241-247.

3. Central Bureau of Statistics - Kenya MoH-K, and ORC Macro - USA. Kenya Demographic and Health Survey, 2003. Calverton, Maryland: CBS, $\mathrm{MOH}$, and ORC, 2004

4. Arudo,J., Gimnig,J.E., TerKuile, F.O., et al. Comparison of government statistics and demographic surveillance to monitor mortality in children less than five years old in rural Western Kenya. Am. J. Trop. Med. Hyg. 2003; 68(SuppI 4): 30-37.

5. Shapiro, R.L., Kumar, L., Phillips-Howard, P., et al. Antimicrobial-resistant bacterial diarrhoea in rural western Kenya. J. Infect. Dis. 2001; 183: 11: 1701-1704.

6. Brooks, J.T., Ochieng, J.B., Kumar, L., et al. Surveillance for bacterial diarrhoea and antimicrobial resistance in rural western Kenya, 1997-2003. Clin. Infect. Dis. 2006; 43:4:393-401.

7. Performance standards for antimicrobial disk diffusion susceptibility tests: 8th informational supplement. NCCLS document M10058. Villanova, PA: National Committee for Clinical Laboratory Standards, 1998.

8. Huang, M.B., Baker, C.N., Banjeree, S. and Tenover, F.C. Accuracy of the $\mathrm{E}$ test for determining antimicrobial susceptibilities of staphylococci, enterococci, campylobacter jejuni, and gram-negative bacteria resistant to antimicrobial agents. J. Clin. Microbiol. 1992; 30:3243- 3248.

9. Ewing, W.H. Identification of Enterobacteriaciae. 4th Edition ed. New York: Elsevier Science Publishing Co. Inc., 1986.

10. World Health Organization. Model chapter for text books. Intergrated management of childhood illness. Available at http://www.who.int/child-adolescent-health/ New- Publications/IMCI/WHO-FCH-CAH-00.yo/WHOFCHL CAH-00. Yo PDF. Accessed. June 17, 2007.

11. Gedlu, E. and Asseffa, A. Campylobacter enteritis among children in north-west Ethiopia: a 1-year prospective study. Ann. Trop. Paediatr. 1996; 16: 207-212.

12. Lindblom, G.B., Ahren, C., Changalucha, J. et al. Campylobacterjejuni/coliand Enterotoxigenic Escherichia coli (ETEC) in faeces from children and adults in Tanzania. Scand. J. Infect. Dis. 1995; 27: 589-593.

13. Bodhidatta, L., Vithayasai, N. Eimpokalarp, B., et al. Bacterial enteric pathogens in children with acute dysentery in Thailand: Increasing importance of quilonone-resistant Campylobacter. Southeast. Asian J. Trop. Med. Pub. Health. 2002; 22: 4:752-757.

14. Megraud, F., Boudraa, G., Bessaoud, K., et al. Incidence of Campylobacter infection in infants in western Algeria and the possible protective role of breast feeding. Epidem. Infect. 1990;105:73-78.

15. Bokkenheuser, V. D., Richardson, N. J., Bryner, J. H., et al. Detection of enteric campylobacteriosis in children. J. Clin. Microbiol. 1979; 9:2:227-232.

16. Simango, C. and Nyahanan, M. Campylobacter enteritis in children in an urban community. Cent Afr. J. Med. 1997; 47: 6:172-175.

17. Tornheim, J.A., Manya, A.S., Oyando, N., et al. The epidemiology of hospitalized pneumonia in rural Kenya: the potential of surveillance data in setting publichealth priorities. Int. J. Infect. Dis. 2007;11:6:536543.

18. Weber, M.W., Milligan, P., Sanneh, M., et al. An epidemiological study of RSV infection in the Gambia. Bull. World. Hlth. Org. 2002; 80: 562-568.

19. Hague, R., Mondal, D., Kirkpatrick, B.D., el al. Epidemiologic and clinical characteristics of acute diarrhoea with emphasis on Entamoeba histolytica infections in preschool children in an urban slum of Dhaka, Bangladesh. Am. J. Trop. Med. Hyg. 2003; 69:398-405.

20. Molbak, K., Jensen, H., Ingholt, L. and Aaby, P. Risk factors for diarrhoeal disease incidence in early childhood: a community cohort study from GuineaBissau. Am. J. Epidemiol. 1997; 146:3:273-282.

21. Osano, O. and Arimi, S.M. Retail poultry and beef as sources of Campylobacter jejuni. East. Afr. Med. J. 1999; 76:141-143.

22. Oberhelman, R. A., Gilman, R. H., Sheen, P. et al. Campylobacter transmission in a Peruvian shantytown: a longitudinal study using strain typing of Campylobacter isolates from chickens and humans in household clusters. J. Infect. Dis. 2003; 187:260-269.

23. Coker, A.O., Isokpehi, R.D., Thomas, B.N., Amisu, K.O. and Obi,C.L.Human campylobacteriosis in developing countries. Emerg. Infect. Dis. 2002; 8:3:237-244. 
24. Brent, A.J., Oundo, J.O., Mwangi, I., et al. Salmonella bacteremia in Kenyan children. Pediatr. Infect. Dis. J. 2006; 25:230-236.

25. Kariuki, S., Revathi, G., Kariuki, N., et al. Characterisation of community acquired nontyphoidal Salmonella from bacteraemia and diarrhoeal infections in children admitted to hospital in Nairobi, Kenya. BMC MicrobioI. 2006;15: 101-111.

26. Prasad, N.R. and Virk, K.J. Malaria as a cause of diarrhoea - a review. PNG Med. J. 1993; 36: 337-341.

27. Karney, W.W. and Tung, J. Malabsorption in Plasmodium falciparum malaria. Am. J. Trop. Med. Hyg. 1972; 21:1-5.
28. Sodemann, M., Jakobsen, M.S., Molbak, K., et al. Malaria parasitemia and childhood diarrhoea a periurban area of Guinea-Bissau. Am. J. Trop. Med. Hyg. 1999; 61:2:336-338.

29. Sodeinde, O., Gbadegesin, R.A., Ademowo, O.G. and Adeyemo, A.A. Lack of association between Falciparum malaria parasitemia and acute diarrhoea in Nigerian children. Am. J. Trop. Med. Hyg. 1997; 57: 702-705.

30. Saidi,S.M., Lijima, Y., Sang, W.K., et al. Epidemiological study on infections diarrhoeal diseases in children in a coastal rural area of Kenya. Microbiol. Immunol. 1997; 41: 773- 778 . 